\title{
The importance of surveying in determining denture design in partially removable dentures
}

\author{
Armawati Arafi ${ }^{1,2^{*}}$, Ike D. Habar
}

\section{Abstract}

Objective: The purpose of making partially removable dentures is for the rehabilitation of tooth loss situations, mastication, esthetics, and improving the health of oral tissues. Some patients are dissatisfied with removable denture, primarily because at the time of functioning the denture becomes unstable; therefore, clinicians are expected to find ways how to improve the denture stability in artificial dentures.

Methods: The references in this review are taken from textbook and internet/Google search using key words out. Full text articles in English that were published from 1981 to 2014 are presented. This review explains about surveying in prosthodontics. Surveyor is a tool for alignment used in making artificial teeth to find and describe the contours and positions of abutment teeth and related structural aspects. The goal is to analyze the model for the best path of insertion selection.

Results: A fundamental requirement in understanding the use of surveyors is to prevent the inappropriateness of the path of insertion. To get a good guide plane, surveying should be done. This guide plane is one of the important factors in determining the path of insertion and the stability of partially removable denture.

Conclusion: The dentist should precisely control the use of surveyors in the manufacture of partially removable dentures. Thus, optimum use of surveyors data will help produce a stable denture and can support the mastication function.
${ }^{1}$ Department of Prosthodontics, Faculty of Dentistry, Hasanuddin University, Makassar, Indonesia ${ }^{2}$ Department of Prosthodontics, Regional Public Hospital H. Padjonga dg. Ngalle Takalar District, South Sulawesi, Indonesia
*Corresponding to: Armawati Arafi, Department of Prosthodontics, Faculty of Dentistry, Hasanuddin University, Makassar, Indonesia armawatinailah@gmail.com

Received: 24 0ctober 2017

Revised: 8 November 2017

Accepted: 9 November 2017

Available online: 1 December 2017

Keywords: Guiding plane, Path of Insertion, Surveying

Cite this Article: Arafi A, Habar ID. 2017. The importance of surveying in determining denture design in partially removable dentures. Journal of Dentomaxillofacial Science 2(3): 147-149. D0I: 10.15562/jdmfs.v2i3.635

\section{Introduction}

Teeth are the main functional component of the oral cavity. Teeth provide various functions, including mastication, speaking and aesthetics. The absence of teeth in the oral cavity can cause difficulties in chewing food, poor speech and aesthetics and greatly affect the quality of life. ${ }^{1}$

The goal of treatment with artificial teeth is to improve the function of mastication, restore aesthetic function, improve phonetic function, and maintain the health of oral tissues of the existing oral cavity. However, patients often feel dissatisfied with the denture when chewing food or talking. This is because the denture is less retentive and unstable. In the manufacture of partially removable dentures, artificial denture instability is partly due to the absence of inappropriate guiding plane when determining the path of insertion, and a good guiding plane can be obtained by conducting a survey. ${ }^{2}$

Dental surveyors can be used to prevent problems arising from the manufacture of partially removable dentures that lack in stability. Many professionals working with oral rehabilitation fail to take advantage of the use of surveyors in planning and designing partial denture chromium and other metal alloy frames. ${ }^{3}$

Partially removable denture placement paths, if guided by a parallel guide plane of each other's teeth, ensure that partially removable partial denture wings fit over the edentulous ridge structure and that the metal framework fits the surface of the guide plane, preventing food from collecting in the empty space between the surface of the metal framework and the intra-oral surface. The cleavage in partially removable denture involves an adequate amount of undercut to obtain better retention in partially removable dentures. ${ }^{4}$

The purpose of writing this article to find out how the use of surveying is very important at the time of manufacture and design of partially removable dentures. This article describes the objectives and benefits of surveyors and surveying steps in obtaining the guide plane and the appropriate path of insertion.

\section{Methods}

Surveying is the first step in designing partially removable dentures. The term survey is defined as the procedure undertaken, "To evaluate conditions, values, or situations, to consider" or "Determine the shape and position of unity in terms of linear and angular measure" or "to see and observe" or as the "Procedure to locate or describe Contours and the position of the supporting tooth and other related structures before designing a partially removable denture." The fourth definition is the most precise: 
surveying is the "Analysis and comparison of the protrusion portions of tooth structure contours associated with partial denture fabrication." Surveying is done using surveyors. ${ }^{5}$ A surveyor is defined as a tool used to determine the alignment of two or more surfaces of the tooth or other portions of the dental arch, which modifies the surface of the tooth, to accommodate the placement of component parts of partial denture in the ideal positioning of tooth, which will help with prognosis. Surveyors are essential for planning, execution and examination of oral changes for partially removable dentures. Although it may not necessarily have an impact on the occlusal rest preparation of the buffer gear, the use of the surveyor is essential for the planning of alteration of all tooth surfaces involved with the support, stabilization and retention of partially removed dentures. ${ }^{6}$

\section{Survey objectives}

The success in designing a partially removable denture with a flexible component depends on the most appropriate position to achieve good and strong resistance. We need to determine the path of artificial tooth insertion so that there is no obstacle when entering through this path; first step is to mark the height of the contour on the area (hard and soft tissue) above the undercut, and then marking the survey line (the height of contours on the teeth) and marking an undesirable undercut where a denture is not allowed to pass through the area. ${ }^{5}$

\section{Types of surveyors}

Surveyors commonly used include the following: Ney surveyor (the most widely used), Jelenko surveyor and Williams surveyor.

The structure of all these surveyors is essentially similar to one another. In general, surveyors from Williams are more flexible. ${ }^{5}$ There are a number of surveyors. Williams surveyor designs vary a lot, but most of them have the parts mentioned below; it has a platform where the model holder can move, a vertical arm that supports the superstructure, a horizontal arm that extends in the right corner of the vertical pole and a surveying arm that extends vertically on the horizontal arm. The surveying tool will be placed on the mandrel and used for various surveying applications. Surveying tools include the following: analyzing rod used to determine the surface alignment of the model, carbon markers used for marking the height of contours on one or more model surfaces, undercut gauge used to identify the undercut position of the model, wax blades used during block-out procedures, and survey tables designed to hold the model. ${ }^{6}$ Because master model surveys include oral preparation, placement paths, retentive location locations, remaining structural disturbance locations should be known before continuing with the final design of the metal frame denture. The purpose of the master model survey is to choose the path that best suits placement and aesthetics, in order to enable the measurement of the retention area and identify the location of the end of the grip on the flexible part of the grip arm used. Flexibility will depend on many of the following factors: the alloy used for the grip, the design and the type of grip, whether the shape is round or semi-round, whether it is from cast or wrought material and the length of the arm grip from the origin to the end; the retention will then depending on flexibility of the arm grip, the size of the undercut and the depth of the grip tip placed into the undercut. To find undesirable undercut areas to be crossed by rigid restoration parts during placement and displacement, these should be eliminated by the block-out, to cut the block-out material parallel to the placement path before duplication. ${ }^{7}$

\section{Guiding plane}

A flat surface in the occlusal-gingival direction of the supporting tooth is considered a guiding plane. Guiding plane is the axial surface of the teeth that contacts the rigid portion of the artificial tooth while attaching and removing the artificial tooth from the mouth. ${ }^{3,8}$

Guiding planes should be made parallel to each other because guiding plane acts as a path of insertion. Guiding plane should be relatively long, in the range of $2-4 \mathrm{~mm}$, in the direction of the occlusal gingiva and contact the rigid portion of the denture. ${ }^{9}$

\section{Path of insertion}

The path of insertion and the loose direction of the partially removable denture should be in the direction in which the artificial tooth moves with respect to the existence of the support system when the removable denture is placed or released from the mouth, guided by the partial contact of the rigid partial tooth with the support. ${ }^{3}$

\section{Discussion}

During the early twentieth century, most of the removable partial dentures were designed and fabricated using many techniques without following common standards. Probably the most common technique is one that involves looking at tooth molds and guessing about the undercut mechanical location. Thus, partially removable denture metal frames need to be designed, manufactured and matched to precise specifications. So as expected, 
ultimately the process of denture fittings in the mouth will take time and often result in incomplete denture. ${ }^{6}$

Determining unwanted undercut location. Any undesirable undercuts should be blocked (filled out) to prevent any obstacles. Block-out is done using night or block-out material. Usually block-out is done in the work model before it is duplicated into a refractory model. The resulting refractory model will be used exclusively for denture casting of some metalstripped skeletons and may not have an undercut, which has previously been blocked on the work model. Undesirable undercuts should be filled (block-out) to the contour elevation according to the tide direction. Contour should be studied using a survey tool such as wax knife to determine the alignment of teeth/guiding plane. The guiding plane that is parallel to supporting teeth must be determined. The path of insertion of partially removable dentures is usually parallel to the buffer axis. Alignment is important to facilitate insertion and release of artificial teeth. If alignment is not obtained, it must be made by contouring the enamel surface or by placing restorations on at least one tooth. Unwanted undercuts on the teeth should be identified and eliminated. Determining the path of artificial tooth insertion, when surveyingto evaluate the alignment of a buffer tooth, the model should tilted to the long axis of the parent gear parallel to the vertical axis. This slope provides an angle for the direction of the denture tide. Tilt model can be done in the direction of anterior, posterior, right, or left positions. The angle of the slope should not exceed $10^{\circ}$. If the slope exceeds $10^{\circ}$, partially removable denture design requires a very wide opening of the mouth for insertion. ${ }^{5}$

\section{Conclusion}

Partially removable dentures should be properly designed so that there be no pressure on the supporting teeth beyond the physiological tolerance, which can easily be placed and discharged by the patient and should be maintained against reasonable amount of forces, in order to avoid an unfavorable appearance.

\section{Conflict of Interest}

The authors report no conflict of interest.

\section{References}

1. Javid YP. Assessment of partially edentulous patients based on kennedy's classification and its relation with gender predilection. International J Scientific Study 2014;2: 32-36.

2. Marion B, Emmanuel N, Marine S, et al. Impact of removable partial denture prosthesis on chewing efficiency. J Appl Oral Sci 2013;21: 392-396.

3. Bezzen OL, Mattos, Ribero. Surveying removable partial dentures: the importance of guiding planes and path of insertion for stability. The J Prosthetic Dentist 1997; 78: 412-418.

4. John SM. The path of palcement of a removable partial denture: a microscope based approach to survey and design. J Adv Prosthodont 2015;7: 76-84.

5. Cotmore JM, E.B.Mingledorf EB, Pomerantz JM, et al. Removable partial denture survey: Clinical practice today. JPD 1983;49: 321-327.

6. Carl FD, Martin AF, Albert DG. The glossary of prosthodontic terms. JPD 1956;117; 1-34.

7. Carr AB, Mcgivney GP, Brown DT. McCracken's removable partial prosthodontics. 12 th ed. Minnesota: Elsevier Mosby; 2010. p. 608.

8. Krikos AA. Preparing guide planes for removable partial dentures. 1975;34: 152-155.

9. John E, Holt DMD. Guiding planes: when and where. JPD 1981;46: 4-6.

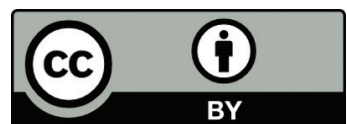

This work is licensed under a Creative Commons Attribution 